\title{
Enhancing HSP70-ShRNA transfection in 22RV1 prostate cancer cells by combination of sonoporation, liposomes and HTERT/CMV chimeric promoter
}

\author{
YUN HUA LI* , LI FANG JIN* , LIAN-FANG DU, QIU SHENG SHI, LONG LIU, \\ XIAO JIA, YING WU, FAN LI and HANG HUI WANG \\ Department of Ultrasound, Shanghai First People's Hospital Affiliated to Shanghai Jiao Tong \\ University School of Medicine, Shanghai 200080, P.R. China \\ Received February 17, 2013; Accepted April 10, 2013
}

DOI: $10.3892 /$ ijo.2013.1921

\begin{abstract}
Gene therapy is a potentially viable approach for treating hormone-refractory prostate cancer (HRPC), it requires efficient delivery systems and a target gene. Inducing carcinoma cell apoptosis by inhibition of heat shock protein 70 (HSP70) overexpression has been emerging as an attractive strategy for cancer therapy. In our study, the high tumor-specificity of human telomerase reverse transcriptase (HTERT) expression prompted the use of an HTERT/cytomegalovirus (CMV) chimeric promoter to drive HSP70-ShRNA expression to induce HRPC 22RV1 cell apoptosis. At the same time, sonoporation induced by ultrasound-targeted microbubble destruction (UTMD) was utilized for delivery of plasmid loaded with HTERT/CMV promoter. Our results indicated the combination of sonoporation, low-dose liposomes and HTERT/CMV chimeric promoter as a delivery system has the potential to promote efficient gene transfer with lower cytotoxicity.
\end{abstract}

\section{Introduction}

Gene therapy is a hopeful therapeutic approach for prostate cancer. HSPs as molecular chaperones and anti-apoptotic proteins are a set of highly conserved proteins (1) which are overexpressed in cancer cells against harmful physiological and environmental insults, the cytoprotective functions of HSPs are necessary to maintain cancer cells survival $(2,3)$.

Correspondence to: Dr Lian-Fang Du, Department of Ultrasound, Shanghai First People's Hospital Affiliated to Shanghai Jiao Tong University School of Medicine, 85 Wu Jin Road, Shanghai 200080, P.R. China

E-mail: du_lianfang@163.com

${ }^{*}$ Contributed equally

Key words: ultrasound-targeted microbubble destruction, HSP70, HTERT, gene therapy, human 22RV1 cells
HSP70 has been shown to block a caspase-independent cell death through its association with apoptosis inducing factor (4). Its overexpression has been shown to increase the tumorigenicity of cancer cells while HSP70 downregulation strongly decreases tumorigenicity $(5,6)$. Thus targeting HSP70 can lead to cell inactivation by permitting programmed cell death. Tissue or cell specific promoters represent one of the main methods of gene targeting. The HTERT promoter has been widely used to drive various apoptotic genes for cancer gene therapy, which is highly active in cancer cells while was very low expression in normal cells or benign tumors (7-9). In our study, targeting HSP70 gene to cancer cells by HTERT/ CMV promoter it was expected that selective expression of HSP70-ShRNA in 22RV1 cells could extensively reduce HSP70 expression without significant toxicity for normal cells.

UTMD technology has been proven as a promising method for gene delivery $(10,11)$. Sonoporation induced by UTMD describes the increase in the permeability of cell membranes, which introduces the genetic material into cells or tissues with high spatial and temporal specificity, but with minimal toxicity (12). In our study, with the help of sonoporation and less quantity of liposomes, the plasmid pSilencer4.1-EGFP loaded with HTERT/CMV and HSP70ShRNA was efficiently and safely tranferred into the 22RV1 cells under our optimized experimental condition. Higher transfection and apoptosis efficiencies revealed that the ShRNA delivery system might be used for cancer therapy research in the future.

\section{Materials and methods}

Construction of plasmids with luciferase reporter gene. The silencing plasmid vector pSilencer4.1-HTERT/CMV-HSP70ShRNA-EGFP was constructed by R\&S Biotechnology Co., Ltd.(Shanghai,China), the other 2 vectors pSilencer4.1-HTERT/ CMV-EGFP and pSilencer4.1-CMV-HSP70-ShRNA-EGFP were also synthesized as control.

A 238-bp core sequence (-217-20 bp upstream, following genebank NM_198253) was the candidate for driving HSP70ShRNA expression and the sequences of HSP70-ShRNA 
were designed as follows according to genebank L12723.1. HSP70-F, 5'-TGCTGACACCAGGCTGGTTGTCAGAAGT TTTGGCCACTGACTGACTTCTGACACAGCCTGGTGT -3'. HSP70-R, 5'-CCTGACACCAGGCTGTGTCAGAAGTC AGTCAGTGGCCAAAACTTCTGACAACCAGCCTGGT GTC -3'.

Cell culture. Human prostate carcinoma cells 22RV1 were maintained in RPMI-1640 medium containing 10\% FBS, penicillin $(100 \mathrm{U} / \mathrm{ml})$ and streptomycin $(100 \mu \mathrm{g} / \mathrm{ml})$ at $37^{\circ} \mathrm{C}$ in a humidified incubator with $5 \% \mathrm{CO}_{2}$. Normal human prostatic epithelial cells RWPE-1 were maintained in a serum-free culture medium K-SFM, cells were purchased from Chinese Academy of Sciences and Cell culture reagents were all purchased from Gibco (USA).

Detection of the plasmid function. In this expriment, 3 vectors were, respectively transfered into 22RV1 and RWPE-1 cells for the verification of their function with the help of Lipofectamine 2000 (Invitrogen, USA). 22RV1 and RWPE-1 cells were plated on a 12 -well plate at a density of $1.5 \times 10^{5}$ cells/ well. After $48 \mathrm{~h}$ of incubation, the medium was replaced by fresh medium RPMI-1640 without FBS or K-SFM, $250 \mathrm{ng}$ (1 $\mu \mathrm{l}$ ) different vector recombinations (replaced by PBS in the control group) and $1 \mu 1$ Lipofectamine 2000 were added to the cells, then the mixture volume per well was supplemented to $250 \mu \mathrm{l}$ with culture medium. After $6 \mathrm{~h}$ of incubation, the mixture was replaced by $1 \mathrm{ml}$ fresh medium RPMI-1640 with $10 \%$ FBS or K-SFM for another $48 \mathrm{~h}$. Fluorescent microscope (Zeiss, Axiovert S100, Germany) was used for observation and quantitative analysis of EGFP expression, and estimated using a FACSCalibur flow cytometer (Becton-Dickinson, Franklin Lakes, NJ, USA).

Real-time PCR. To establish silencing effect of different vectors at the HSP70 mRNA level, a real-time PCR analysis of 22RV1 cells was carried out. The total RNA was extracted from the different groups after $48 \mathrm{~h}$ of transfection. Then, reverse transcription to synthesize cDNA was achieved using First Strand cDNA Synthesis kit (Takara, Tokyo, Japan). Real-time PCR was performed with cDNA by using the SYBR $^{\circledR}$ Premix Ex Taq ${ }^{\mathrm{TM}}$ kit (Takara). The final results were evaluated by $2^{-\triangle \Delta C T}$ analytical method. The PCR primer sequences are as follows: HSP70-F, 5'-TACTGTGGACCTGC CAATCG-3'. HSP70-R, 5'-CATTCCGCTCCTTCTCCA GTT-3'. Internal standardization, GAPDH-F, 5'-CTTAGCAC CCCTGGCCAAG-3'. GAPDH-R, 5'-GATGTTCTGGAGA GCCCCG-3'.

\section{In vitro study}

Cell culture. 22RV1 cells were maintained and prepared as descibed above.

Ultrasound exposure protocol. A therapeutic US machine (Physiomed, Erlangen, Germany) was used, the area of the probe (1 MHZ) was $\sim 5 \mathrm{~cm}^{2}$. The groups were exposed to optimized ultrasound conditions (power, $0.8 \mathrm{~W} / \mathrm{cm}^{2} ; 20 \%$ duty cycle; exposure time, $1 \mathrm{~min}$ ). The SonoVue powder (Bracco, Milan, Italy) was mixed with $5 \mathrm{ml}$ saline. After shaking for $30 \mathrm{sec}$, white galactoid microbubble suspension was prepared, which was added into the different groups for simultaneous ultrasound irradiation.

Experimental groups. After the function of pSilencer4.1HTERT/CMV-HSP70-ShRNA-EGFP was verified, 22RV1 cells were divided into the following 5 groups for gene transfection experiment. The control group a, 22RV1 cells with $52 \mu \mathrm{l}$ PBS; the control group b, 22RV1 cells with $1 \mu 1$ plasmid $+51 \mu$ l PBS; the control group c, 22RV1 cells with $1 \mu 1$ plasmid $+1 \mu 1$ Lipo $2000+50 \mu 1$ PBS; the control group d, 22RV1 cells with $1 \mu \mathrm{l}$ plasmid $+1 \mu \mathrm{l} \mathrm{PBS}+50 \mu \mathrm{l}$ SonoVue microbubble + ultrasound; the test group e, 22RV1 cells with $1 \mu$ l plasmid $+1 \mu 1$ Lipo $2000+50 \mu 1$ SonoVue microbubble + ultrasound. Then the mixture volume per well was supplemented to $1 \mathrm{ml}$ with culture medium and the final concentration of the microbubble was adjusted to $20 \%$ in the groups. All experiments were carried out in triplicate.

Transfection. We compared the cellular uptake efficiencies of different cell groups. The tranfection experiment was carried out. After $6 \mathrm{~h}$ of different mixture incubation, the mixture was replaced by $1 \mathrm{ml}$ fresh medium RPMI-1640 with 10\% FBS for another $48 \mathrm{~h}$. Fluorescent microscope and FACSCalibur flow cytometer was used for evaluation of EGFP expression.

Detection of apoptosis by flow cytometry. Phosphatidylserine (PS) externalization is one of the main event during cell early apoptosis. To detect PS externalization, transfected cells were harvested by trypsinization and washed twice with PBS after $48 \mathrm{~h}$ of transfection. Washed cells were resuspended in $200 \mu \mathrm{l}$ binding buffer. Annexin V-PE was added according to the manufacturer's instructions (Biosea Biotechnology Co., Ltd., Beijing, China). After incubation for $20 \mathrm{~min}$ at room temperature, $400 \mu \mathrm{l}$ binding buffer was added and samples were immediately analysed on a FACSCalibur flow cytometer (Becton-Dickinson) with excitation using a 495-nm argon ion laser.

Real-time PCR. To compare silencing effect of the 5 groups at the HSP70 mRNA level, a real-time PCR analysis of 22RV1 cells was carried out after $48 \mathrm{~h}$ of transfection, the methods was as stated above.

Western blot analysis. Total protein extracts of each cell group were resolved by $10 \%$ SDS-PAGE and transferred on PVDF (Millipore) membranes. After blocking, the PVDF membranes were washed 3 times for 10 min with TBST at room temperature and incubated with primary antibody (rabbit anti-human HSP70 and caspase-3 antibody; 1:1,000, Epitomics, USA). Following extensive washing, membranes were incubated with secondary peroxidase-linked goat anti-rabbit IgG $(1: 5,000$, Epitomics) for $2 \mathrm{~h}$. After washing 3 times for $10 \mathrm{~min}$ with TBST at room temperature once more, the immunoreactivity was visualized by ECL kit (Biosea) and membranes were exposed to Kodak XAR-5 film (Sigma-Aldrich, USA).

Cell toxicity assay. 22RV1 cells were plated on a 96-well plate at a density of $8 \times 10^{3}$ cells/well and divided into 5 groups after $24 \mathrm{~h}$ of incubation. The control plasmid pSilencer4.1-HTERT/ CMV-EGFP was selected to avoid ShRNA silencing effect. We 

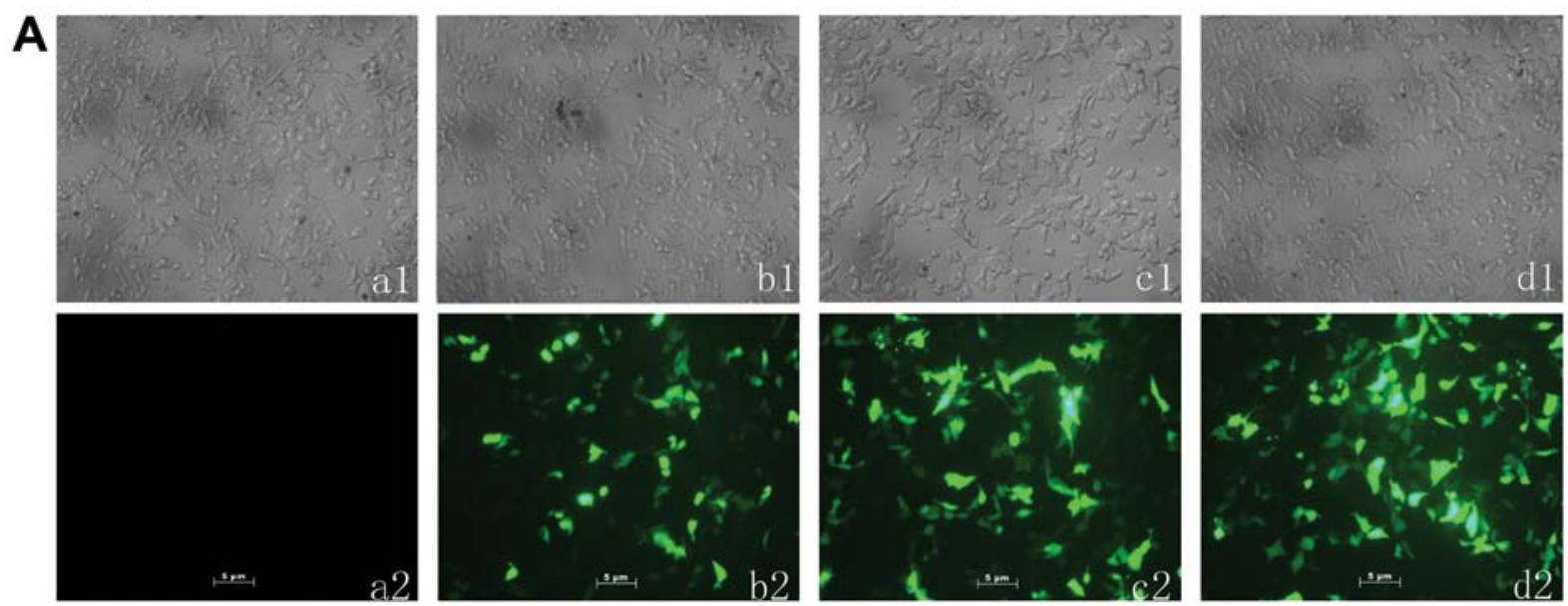

(x200)
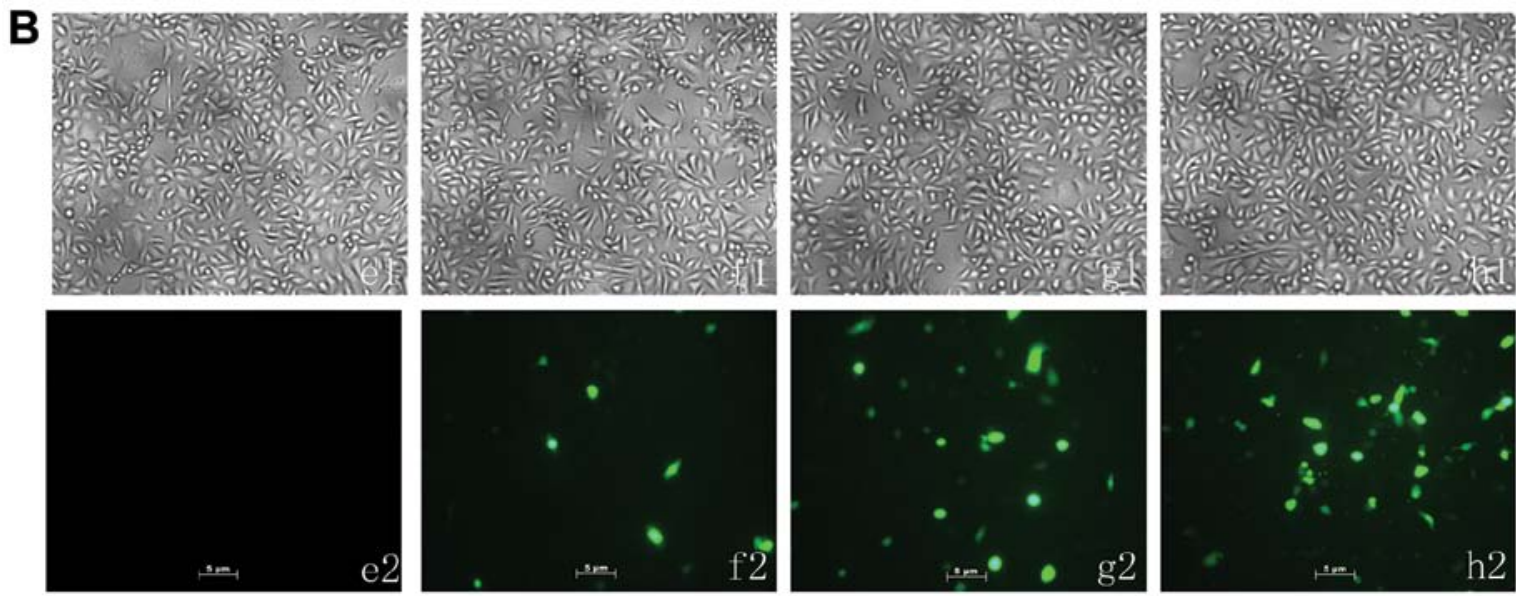

(x200)

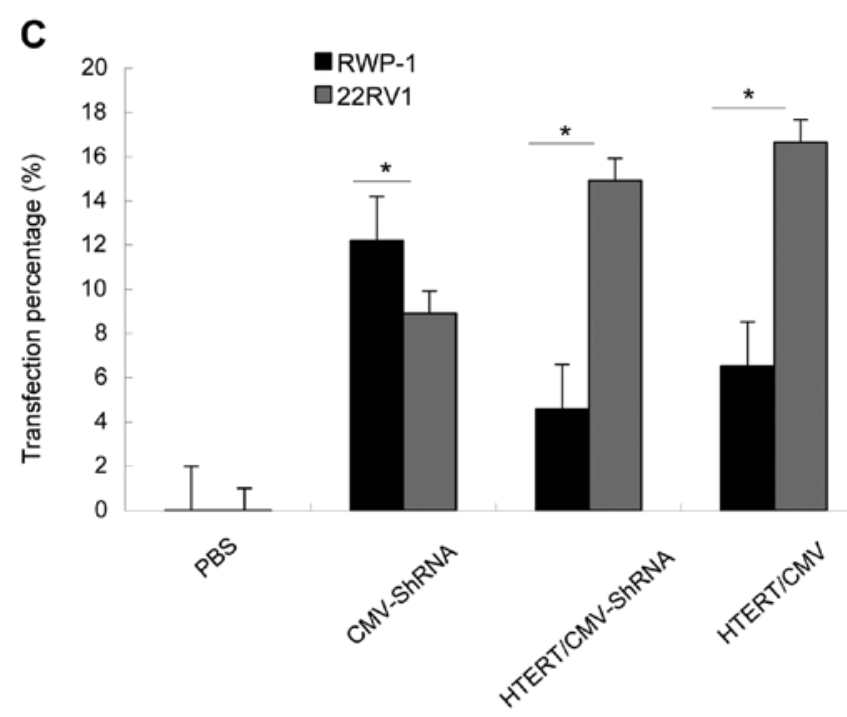

Figure 1. Intracellular uptake of different plasmid recombinations after $48 \mathrm{~h}$ of transfection. (A) Panel 1, white light images of 22RV1; panel 2, a2, b2, c2 and d2, fluorescent images of PBS, CMV-ShRNA, HTERT/CMVShRNA, HTERT/CMV groups. (B) Panel 1, white light images of RWPE-1; e2, f2, g2 and h2, fluorescent images of PBS, HTERT/CMV-ShRNA, HTERT/CMV, CMV-ShRNA groups. (C) Comparison of mean transfection percentage of 8 groups using flow cytometry. The quantity of fluorescence in HTERT/CMV group was the highest for 22RV1; CMV-ShRNA group was the highest for RWPE-1 ( $\left.{ }^{*} \mathrm{p}<0.05\right)$, while there was no statistical difference between HTERT/CMV group and HTERT/CMV-ShRNA for 22RV1 and RWPE-1.

performed CCK 8 test $2 \mathrm{~h}$ after cells were differently treated to measure the transient cytotoxicity of UTMD and less quantity of Lipofectamine 2000. CCK8 solution $(10 \mu \mathrm{l})$ was added to $100 \mu \mathrm{l}$ fresh culture medium and incubated for an additional $4 \mathrm{~h}$, the absorbance was determined at $450 \mathrm{~nm}$ wavelength. Cell Counting kit-8 was purchased from Dojindo (Japan).

\section{In vivo studies}

Animal preparation. To further determine the role of this delivery system, animal experiments were carried out. Untransfected 22RV1 cells were trypsinized and suspended in PBS $\left(5 \times 10^{6}\right.$ cells per $\left.100 \mu \mathrm{l}\right)$ and then inoculated subcutaneously into the right flank of Balb/c female nude mice. Tumor formations were observed in nude mice 2 weeks after injection.

All animals were treated, maintained and sacrificed in accordance with the policies stated in the ARVO Statement for the Use of Animals in Ophthalmic and Vision Research and with the guidelines approved by national and local institutions. When the average tumor diameter reached $5 \mathrm{~mm}$, mice were randomly divided into 5 groups $(\mathrm{n}=8$ per group) and were treated in the following groups: the control group a, tumor injected with $100 \mu \mathrm{l}$ PBS; the control group b, tumor with $20 \mu \mathrm{l}(10 \mu \mathrm{g})$ plasmid $+80 \mu \mathrm{l} \mathrm{PBS}$; the control group c, tumor with $20 \mu \mathrm{l}$ plasmid $+5 \mu \mathrm{l}$ Lipo $2000+75 \mu \mathrm{l}$ PBS; the control group d, tumor with $20 \mu \mathrm{l}$ plasmid $+55 \mu \mathrm{l}$ 


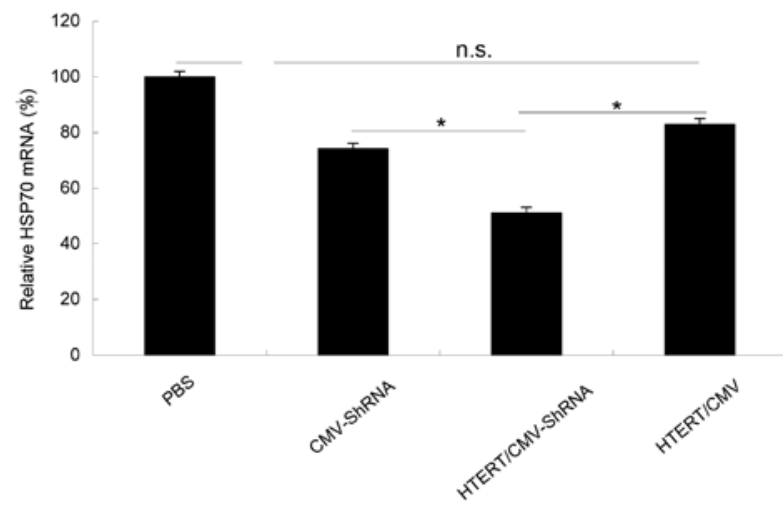

Figure 2. Real-time PCR analysis for relative HSP70 mRNA level of 22RV1 treated with different plasmids after $48 \mathrm{~h}$ of transfection, the HSP70 mRNA level of HTERT/CMV-ShRNA group was the lowest ("p<0.05), there was no difference between PBS group and HTERT/CMV group (ns, not significant).

PBS $+25 \mu 1$ microbubble + ultrasound; the test group e, tumor with $20 \mu 1$ plasmid $+5 \mu 1$ Lipo $2000+25 \mu 1$ microbubble + ultrasound $+50 \mu$ l PBS.

The groups were exposed to optimized ultrasound conditions (power, $2.0 \mathrm{~W} / \mathrm{cm}^{2} ; 20 \%$ duty cycle; exposure time, $2 \mathrm{~min}$ ). Half of the mice were sacrificed on day 2 after being treated and the tumors were removed. Half of the mice were differently treated for the second time 3 days after the first injection and sacrificed 3 weeks later. The short and long diameters of the tumors were measured and tumor volumes $\left(\mathrm{mm}^{3}\right)$ were calculated by the formula: tumor volume $=$ width $^{2} \mathrm{x}$ length $\mathrm{x} 0.52$.

Morphological evaluation. Sections of each tumor were collected and fixed in the glutaraldehyde. The ultrastructure variation of the treated tumors was observed using transmission electron microscopy (Hitachi H-600, Japan) at Shanghai Medical College of Shanghai Jiao Tong University.
Immunohistochemistry. Sections of each tumor were fixed in $4 \%$ paraformaldehyde and embedded in paraffin blocks. Sections $(4 \mu \mathrm{m})$ were used for immunohistochemical examination. HSP70 and caspase-3 protein expression of tumor tissues were detected using standard avidin-biotin immunohistochemical techniques with use of anti-HSP70 and anti-caspase-3 antibody (1:200, Epitomics) according to the manufacturer's instructions. The staining was observed by microscopy (Zeiss, Axioplan 2 Imaging). The result was quantitatively analyzed by the Image-Pro Plus Analysis system (Olympus, Tokyo, Japan) from at least five high power fields. Relative protein expresstion level was evaluated with the average value of optical density (IOD)/field area.

\section{Results}

Detection of the plasmid function. Fig. 1A and B showed the fluorescent images of cellular uptake of different plasmid recombinations in 22RV1 and RWPE-1. Examination with flow cytometer showed pSilencer4.1-HTERT/CMV-EGFP had maximal transfection percentage $(16.66 \pm 0.73)$ and little higher than pSilencer4.1-HTERT/CMV-HSP70-ShRNA-EGFP $(14.92 \pm 0.70)(\mathrm{p}>0.05)$ for $22 \mathrm{RV} 1$ cells, while the 2 plasmids showed notable difference, respectively, when compared with RWPE-1 groups $(6.53 \pm 0.68,4.6 \pm 0.71)(\mathrm{p}<0.05)$ (Fig. 1C), for RWPE-1 cells, pSilencer4.1-CMV-HSP70-ShRNA-EGFP had maximal transfection percentage (12.22 \pm 0.73$)$.

To establish the inhibition effect of HSP70-ShRNA for 22RV1, relative mRNA expression levels were evaluated after transfection. The results showed HTERT/CMV-ShRNA group ranked bottom with $51.2 \%$ (Fig. 2) and CMV-ShRNA group next to it with $74.2 \%(\mathrm{p}<0.05)$. The other control groups showed no significant inhibition effects as compared to test samples. All the results showed the plasmid pSilencer4.1-HTERT/ CMV-HSP70-ShRNA-EGFP was successfully constructed, HSP70-ShRNA silencing function was verified and HTERT/ CMV chimeric promoter has higher transcriptional activity in the 22RV1 tumor cells than telomerase-negative RWPE-1 cells.
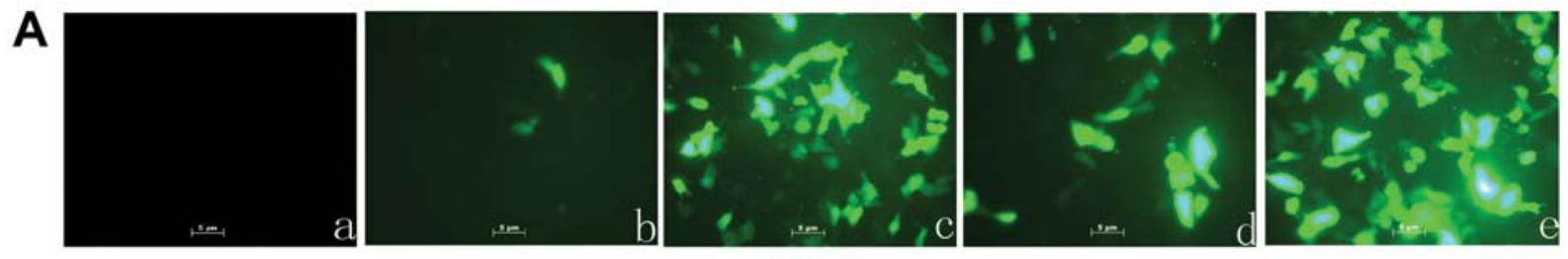

$(x 400)$

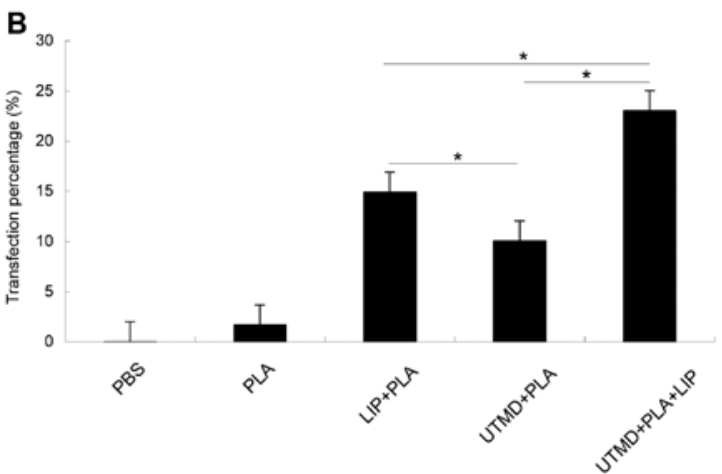

Figure 3. Intracellular uptake of HTERT/CMV-ShRNA plasmid vector mediated by different methods after $48 \mathrm{~h}$ of transfection. (A) Fluorescent images of 5 groups under fluorescent microscope. (B) Comparison of mean transfection percentage of the 5 groups using flow cytometry. There was significant diference between each other $(* \mathrm{p}<0.05)$. 


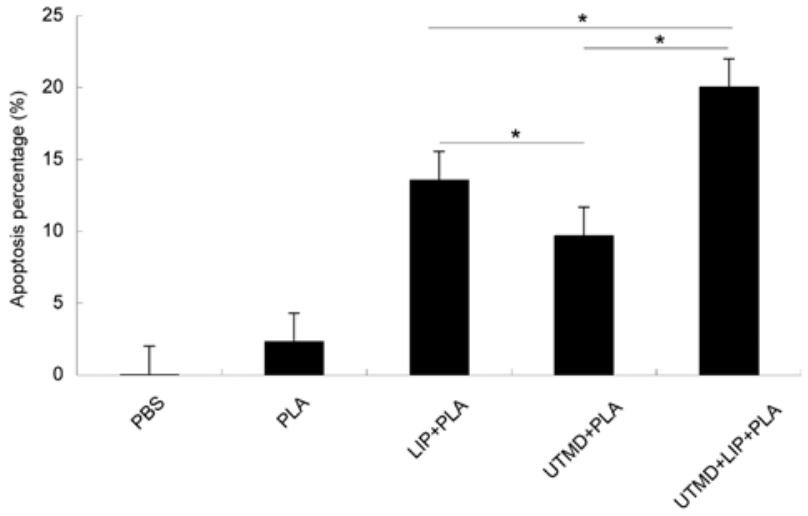

Figure 4. Apoptosis analysis of 12 groups by flow cytometry after $48 \mathrm{~h}$ of transfection. Apoptosis percentage of UTMD+LIP+PLA group was significantly higher than the control groups ( $(\mathrm{p}<0.05)$, while there was no difference between PBS goup and PLA group.

\section{In vitro study}

Transfection and apoptosis percentage by flow cytometry. Fig. 3 showed the UTMD+LIP+PLA group had maximal transfection percentage $(23.05 \pm 0.83)$ and it was significantly higher than LIP+PLA group $(14.92 \pm 0.61)$ and UTMD+PLA group (10.07 \pm 0.74$)$.

In accordance with the results of transfection analysis, the apoptosis percentage of the test group was the highest (20.02\%) and the other 3 groups had significant difference between each other $(\mathrm{p}<0.05)$, while PBS group and plasmid group made no difference $(\mathrm{p}<0.25)$ (Fig. 4). It seems that the UTMD+ LIP could serve as a gene delivery system and scilencing HSP70 expression induced cell apoptosis.

Real-time PCR. To compare the silencing effect of HSP70ShRNA of different groups, relative mRNA expression levels was also examined, HSP70 gene expression of the test group was the lowest with $38 \%$ among all the groups, significantly lower than LIP+PLA group (51.2\%) and UTMD+PLA group $(79 \%)(\mathrm{p}<0.05)($ Fig. 5).

Western blot analysis. HSP70 and caspase-3 protein levels were measured in 22RV1 cells at $48 \mathrm{~h}$ post-transfection by western blot assay (Fig. 6), the results revealed that the expression level of HSP70 protein in cells treated with UTMD+LIP+PLA system was significantly lower than the other groups, while the expression level of caspase- 3 in this group was maximal, therefore, expression level of the 2 proteins presented negative correlation in our experiment.

Cell toxicity assay. Although UTMD techonology has been considered to assist the delivery of molecules into a cell by transiently increasing the membrane and cytotoxicity of Lipo 2000 was confirmed by relative studies, under our optimized condition, cell viability showed no significant difference between PBS group (83.3\%) and test group (81.7\%) $(\mathrm{p}<0.5)$ (Fig. 7). The results demonstrated cell viability could not be injured by UTMD or low quantity of Lipo 2000 .

In vivo studies. Tumor measurement. Fig. 8 shows nude mice with tumor formation with different administrations. Tumor

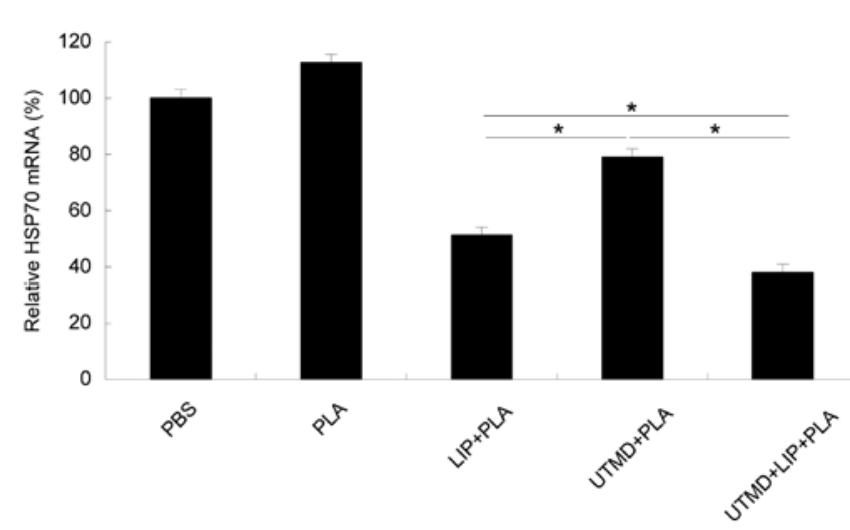

Figure 5. Real-time PCR analysis for relative HSP70 mRNA level of 22RV1 with different treatments. HSP70 mRNA level of UTMD+LIP+PLA group was significantly higher than the UTMD+PLA group and LIP+PLA group $\left({ }^{*} \mathrm{p}<0.05\right)$.

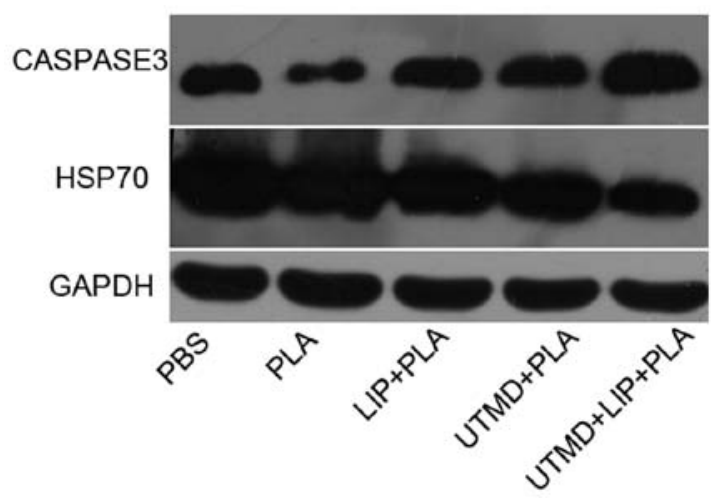

Figure 6. HSP70 and caspase-3 protein levels were measured in 22RV1 cells at $48 \mathrm{~h}$ post-transfection by western blot assays, expression level of the 2 proteins presented negative correlation in different groups, which is obvious in the PBS group and UTMD+LIP+PLA group.

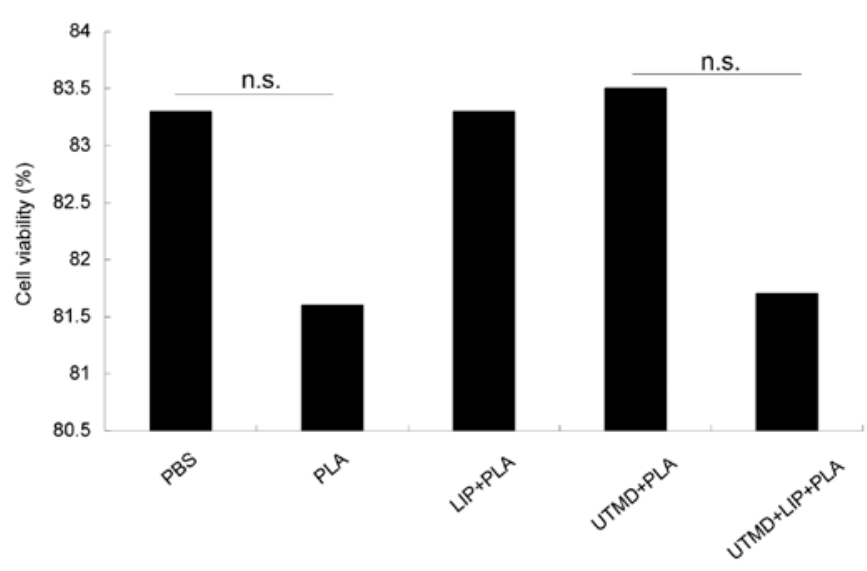

Figure 7. Cell viability assay was carried out using CCK8 test after cells were differently treated, the 5 groups showed no significant difference compared to each other.

formation was observed subcutaneously in all nude mice at 2 weeks after inoculation. During a 5-week follow-up period, it was observed that the tumor volumes were increasing to varying degree for different groups. At 5 weeks, the volume of tumors were the smallest in the test group with $111 \mathrm{~mm}^{3}$ as 
A
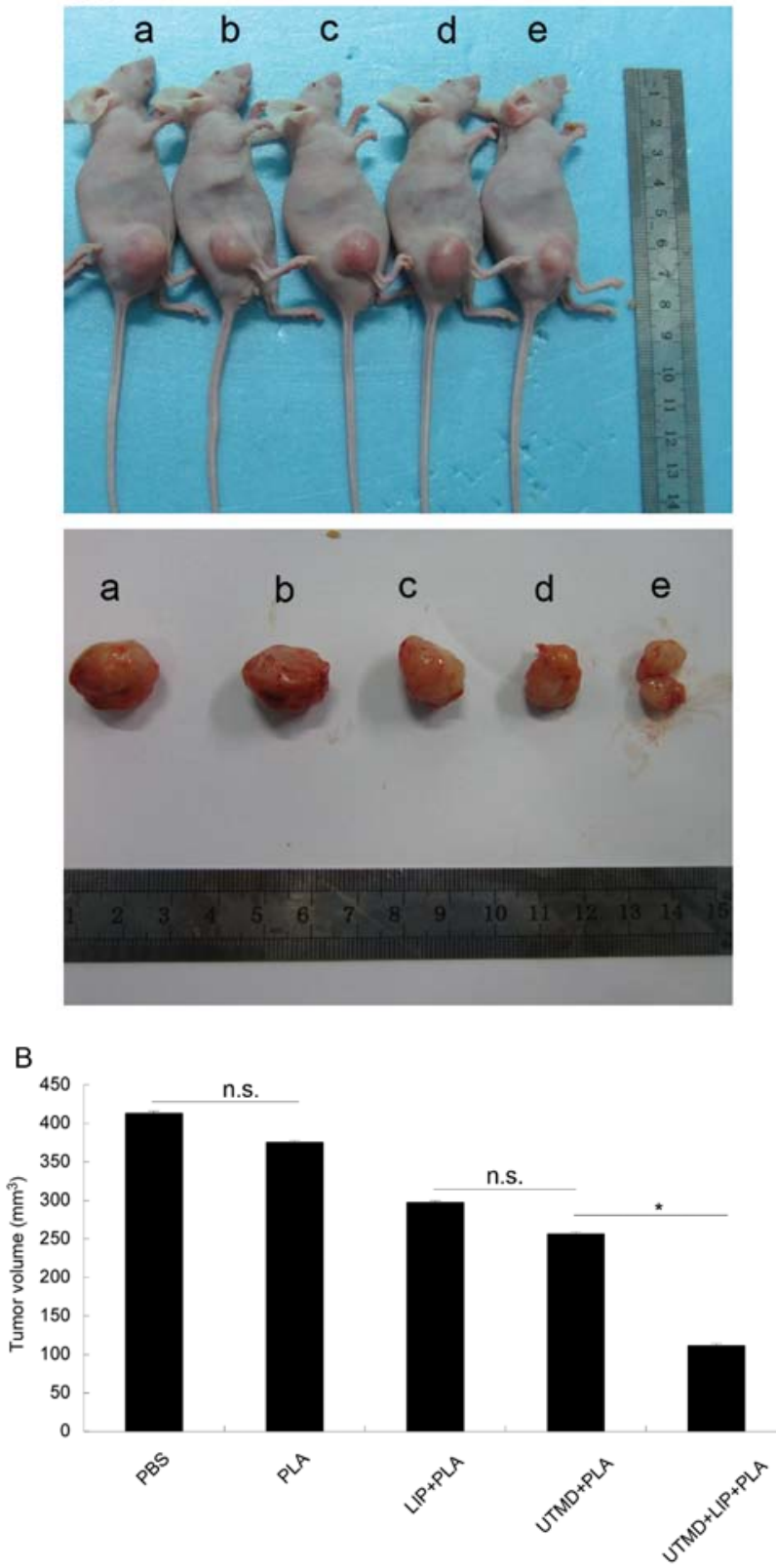

Figure 8. Different treatments suppressed the growth of established 22RV1 tumor xenografts variably in nude mice; images a-e, were consistent with the grouping a-e. (A) The nude mice with tumor formations with different administration; at 3 weeks after the second injection, tumors were dissected and tumor volumes were measured serially. (B) The mean volume of tumors $\left(111 \mathrm{~mm}^{3}\right)$ in the test group (e) was also significantly less than the other groups (ns, not significant; " $\mathrm{p}<0.05$ ). compared with other groups $(\mathrm{p}<0.05)$, while the mean volume was the largest in the PBS group with $435 \mathrm{~mm}^{3}$.

Immunohistochemistry. HSP70 and caspase-3 expression of tumors was detected by immunohistochemical test (Fig. 9). Caspase-3 protein expression occurred in the plasma of the tumor cells, which was expressed as apoptosis index, while HSP70 protein expression in this experiment occurred in the cell membrane and plasma.

Compared with the control groups, HSP70 expression of the UTMD+LIP+PLA group was only $25 \%$ of the PBS group, which demonstrated HSP70 expression was dramatically inhibited $(\mathrm{p}<0.01)$. However, the difference of the 4 control groups between each other was not significant. For caspase-3, an important apoptotic protein, each group exhibited varying degrees of caspase-3 staining positivity. The apoptotic indexes (IOD/field area) were 1.79 \pm 2.29 (group a), 2.15 \pm 3.34 (group b), 14.1 \pm 9.34 (group c), 18.3 \pm 4.29 (group d), 25.76 \pm 5.64 (test group e), respectively. The protein levels of caspase-3 in UTMD+LIP+PLA group significantly increased in tumor tissues $(p<0.01)$.

Morphometric analysis by TEM. After $48 \mathrm{~h}$ of different tumor treatment, apoptotic features (such as condensation of the nuclear chromatin, wrinkling of nuclear membranes, dilation of endoplasmic reticulum, budding and the formation of the apoptosis bodies) were observed under TEM (Fig. 10c-e). It was rare to find an apoptotic cell in group a and b, however, a great number of tumor cells were apoptotic in the test group e, much more than control group $\mathrm{c}$ and $\mathrm{d}$.

Statistical analysis. Data were expressed as the means and standard deviation (mean $\pm \mathrm{SD}$ ). Independent samples t-test was used to determine the significance of the difference between two groups. ANOVA (analysis of variance) was used to determine the significance of the differences in multiple comparisons. Differences were considered significant at $\mathrm{p}<0.05$. Statistical analysis were performed with a software package (SPSS, version 13.0; SPSS, Chicago, IL, USA).

Conclusions. UTMD techonology was expected to significantly facilitate gene transfection by the transient increase of cell membrane permeability. Our study indicated that UTMD exerted a significant enhancing effect on gene transfection in 22RV1 cells with the help of liposome, while UTMD alone played a weak role in increasing gene transfection efficiency.
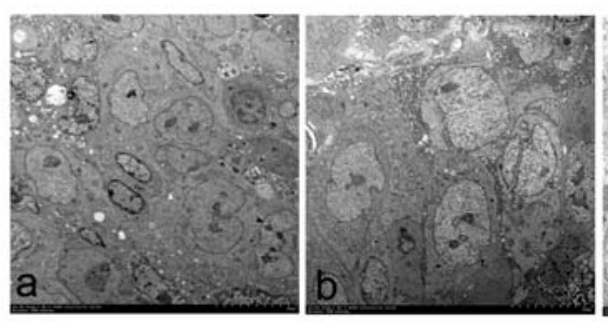
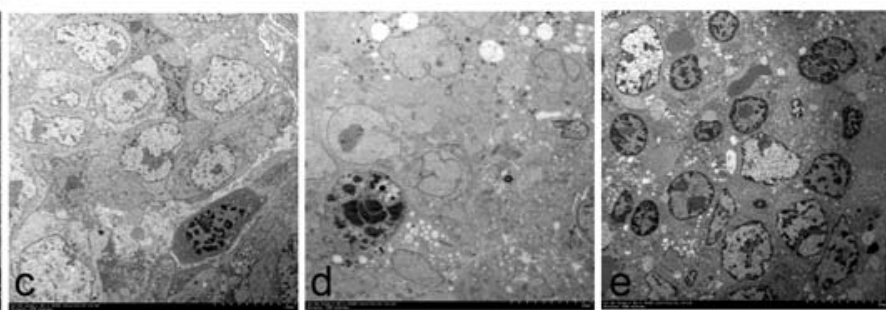

(x7500)

Figure 9. The morphologic changes of tumor tissues observed by TEM. Tumor cells display normal nuclei and endoplasmic reticula in groups a and b; groups $\mathrm{c}$ and d, show slight cell degeneration, with cell volume enlargement, chromatin margination and swelling of mitochondria-like vacuoles; group e, showed more cell degeneration and necrosis, typical apoptotic characteristics were clear under TEM. 

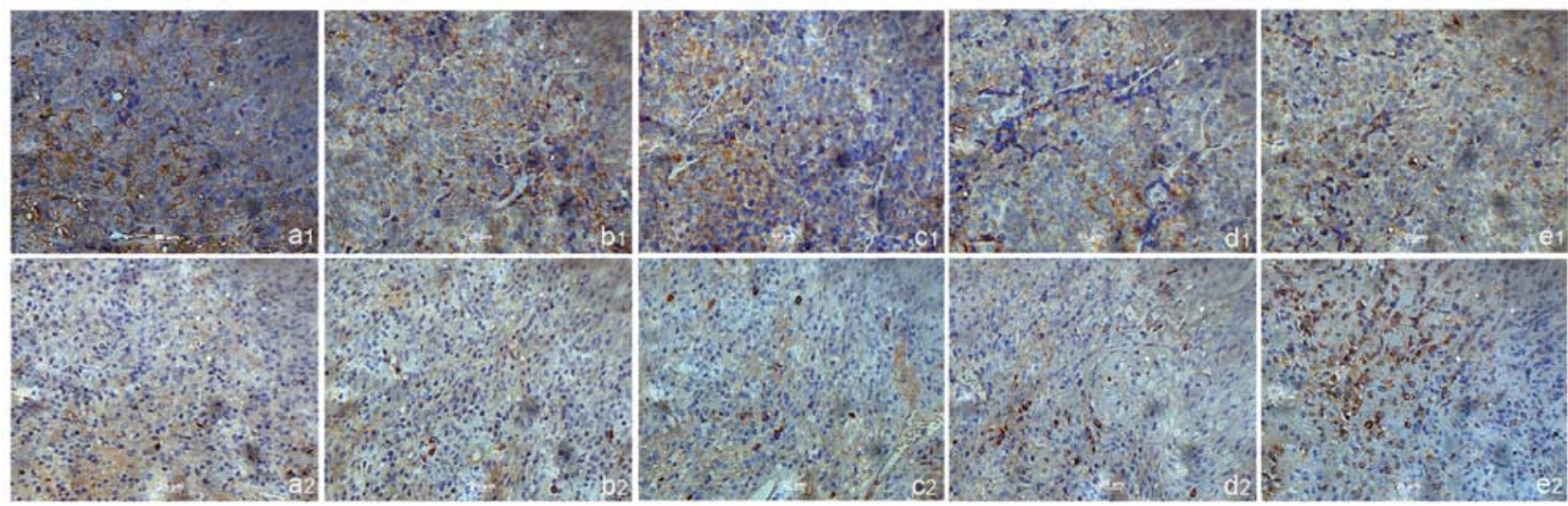

$(x 400)$

Figure 10. HSP70 expression (panel 1) and caspase-3 (panel 2) of tumors were detected by immunohistochemical assays. HSP70 positive staining showed brownish yellow mainly in the cell membrane and were not different among the 4 control groups; while caspase-3 positive staining was brown-red in the cytoplasm, as compared with the control groups, the expression level of HSP70 and caspase-3 in the test group e ranked the lowest and the highest, respectively.

The results indicated that small quantity of liposomes combined with UTMD and special promoter HTERT could synergistically serve as the targeted non-viral gene delivery system without notable cell toxicity. In conclusion, repeated experiments are necessary to verify the feasibility of this novel gene delivery system and further study on the mechanism of sonoporation is needed if UTMD technology is to be properly adopted in the future.

\section{Discussion}

Until recently, there were limited treatment options for hormone-refractory prostate cancer (13). Gene therapy is a promising and exciting breakthrough in the field of prostate cancer. As a member of the HSP family, HSP70 has been considered as cancer relevant protein and its overexpression has been correlated with poor prognosis and resistance to therapy. HRPC cells 22RV1 and ShRNA targeted HSP70 gene were selected in our study. The high tumor-specificity HTERT promoter was also selected to specially and safely enhance transfection efficiency in our study.

The plasmid loaded with HSP70-ShRNA and HTERT/ CMV promoter was delivered into the cell plasma by the sonoporation induced by UTMD combined with liposome, which was expected to achieve downregulation of HSP70 gene expression, to inhibit tumor cell proliferation and to induce tumor cell apoptosis. Cytotoxicity of liposome has been verified for interrupting the physiological process (14). Therefore, only $25 \%$ of recommended dosage was used in this experiment and cell damage was not significant.

In this study, we successfully constructed the recombinant plasmid vector pSilencer4.1-HTERT/CMV- HSP70-ShRNAEGFP. Real-time PCR (for the mRNA level) was carried out to identify the direct inhibitory effect of HSP70-ShRNA. The results showed HSP70-mRNA decreased significantly compared to control groups.

Detection of transfection percentage by flow cytometry demonstrated the HTERT/CMV chimeric promoter remakably improve the gene delivery efficiencies in 22RV1 while failed to exceed the promoter CMV in RWPE-1, which indi- cated HTERT promoter had powerful transcriptional activity and had targeting effect in cancer cells as previously reported $(15,16)$. The gene delivery system driven by tumor-specific promoter HTERT has become a promising modality in cancer therapy (17).

When the plasmid vector was successfully constructed, UTMD technology combined with liposome was desired to further improve the gene delivery efficiency, 5 groups were designed for this experiment. The transfection percentage of the UTMD+LIP+PLA group was the highest and almost 1 time higher than LIP+PLA group and UTMD+PLA group by flow cytometer analysis, which was in line with the results of real-time PCR. At the same time, detection of apoptosis by flow cytometry revealed apoptosis percentage for the test goup was significantly higher than the other control groups, which was also consistent with western blot analysis for caspase-3 protein, which was upregulated in response to HSP70 silencing as apoptotic stimuli. Caspase-3 as an important apoptosis hallmark is well known from many previous studies $(18,19)$. Also in our study HSP70 silencing upregulated caspase-3 expression and hence promoted 22RV1 cell apoptosis (20). Real-time PCR and western blotting showed HSP70-mRNA and protein expression were maximally silenced by HSP70ShRNA in the experimental group. In conclusion, the HSP70 and caspase-3 expression level in the UTMD+LIP+PLA group was the lowest and the highest, respectively, reflecting that the lower dose liposome combined with UTMD was an effective method to facilitate gene delivery.

Sonoporation induced by UTMD promotes the transient and reversible increase in the permeability of cell membranes, it has been proven to enhance gene transfer and could serve as a potential site-specific gene transfer modality (21).

Additonally for liposomes, they bind and condense DNA spontaneously to form complexes with high affinity to cell membranes, endocytosis of the complexes followed by disruption of the endosomal membrane appears to be the major mechanism of gene delivery $(22,23)$. However, many studies have indicated side effects of inertial cavitation induced by UTMD, such as cell apoptosis and cell lysis (24), capillary rupture (25) and hemolysis (26). There was also cytotoxicity 
for liposomes, including inhibition of the ATPase activity (27) and interaction with mitochondrial membrane (28). In our study, optimized UTMD and small quatities of liposome did not induce significant cell viability decrease as evaluated by CCK8 test as compared with PBS group. We obtained some results with statistical significance, however, from the perspective of absolute value, increased range was extremely limited and it was difficult for UTMD alone to exceed the liposome alone at promoting transfection. The mechanism needs to be clarified for UTMD technology to serve reseacher better. Many studies focused on the mechanism and many hypotheses have been formed, but the facts still remain unknown $(12,29)$. Simple animal experiments were carried out to further evaluate the feasibility of this delivery system. The apoptotic characteristics of tumor cells under TEM and the expression level change of HSP70 and caspase- 3 revealed HSP70-ShRNA silencing mediated by this delivery system could inhibit tumor growth and promote apoptosis. Therefore, this multi-targeted gene delivery system was capable of significant growth inhibition in vitro and tumor inhibition in vivo.

\section{Acknowledgements}

This study was supported by the National Natural Science Foundation of China (nos. 81171352/H1805, 81271596/H1805 and nos. 81000617 and 81000687) and Shang Hai Municipal Science and Technology Committee (124119a5300).

\section{References}

1. Calderwood SK, Khaleque MA, Sawyer DB and Ciocca DR: Heat shock proteins in cancer: chaperones of tumorigenesis. Trends Biochem Sci 31: 164-172, 2006.

2. Gurbuxani S, Schmitt E, Cande C, et al: Heat shock protein 70 binding inhibits the nuclear import of apoptosis-inducing factor. Oncogene 22: 6669-6678, 2003.

3. Garrido C, Brunet M, Didelot C, Zermati Y, Schmitt E and Kroemer G: Heat shock proteins 27 and 70: anti-apoptotic proteins with tumorigenic properties. Cell Cycle 5: 2592-2601, 2006.

4. Ravagnan L, Gurbuxani S, Susin SA, et al: Heat-shock protein 70 antagonizes apoptosis-inducing factor. Nat Cell Biol 3: 839-843, 2001 .

5. Nylandsted J, Brand K and Jaattela M: Heat shock protein 70 is required for the survival of cancer cells. Ann NY Acad Sci 926 $122-125,2000$

6. Jego G, Hazoume A, Seigneuric R and Garrido C: Targeting heat shock proteins in cancer. Cancer Lett: Nov 13, 2010 (Epub ahead of print).

7. Ponnala S, Chetty C, Veeravalli KK, Dinh DH, Klopfenstein JD and Rao JS: MMP-9 silencing regulates hTERT expression via betal integrin-mediated FAK signaling and induces senescence in glioma xenograft cells. Cell Signal 23: 2065-2075, 2011.

8. Shen CX, Wen Z, Qian YH, Mu SF and Guan XF: Targeted gene therapy of nasopharyngeal cancer in vitro and in vivo by enhanced thymidine kinase expression driven by human TERT promoter and CMV enhancer. J Exp Clin Cancer Res 29: 94 2010.

9. Li JT, Bian K, Zhang AL, Kim DH, et al: Targeting different types of human meningioma and glioma cells using a novel adenoviral vector expressing GFP-TRAIL fusion protein from hTERT promoter. Cancer Cell Int 11: 35, 2011.
10. Zheng MM, Zhou XY, Wang LP and Wang ZG: Experimental research of RB94 gene transfection into retinoblastoma cells using ultrasound-targeted microbubble destruction. Ultrasound Med Biol 38: 1058-1066, 2012.

11. Li HL, Zheng XZ, Wang HP, Li F, Wu Y and Du LF: Ultrasoundtargeted microbubble destruction enhances AAV-mediated gene transfection in human RPE cells in vitro and rat retina in vivo. Gene Ther 16: 1146-1153, 2009.

12. Park J, Fan Z and Deng CX: Effects of shear stress cultivation on cell membrane disruption and intracellular calcium concentration in sonoporation of endothelial cells. J Biomech 44: 164-169, 2011.

13. Brawley OW: Trends in prostate cancer in the United States. J Natl Cancer Inst Monogr 2012: 152-156, 2012.

14. Ewert K, Slack NL, Ahmad A, et al: Cationic lipid-DNA complexes for gene therapy: understanding the relationship between complex structure and gene delivery pathways at the molecular level. Curr Med Chem 11: 133-149, 2004.

15. Onimaru M, Ohuchida K, Mizumoto K, et al: hTERT-promoterdependent oncolytic adenovirus enhances the transduction and therapeutic efficacy of replication-defective adenovirus vectors in pancreatic cancer cells. Cancer Sci 101: 735-742, 2010.

16. Braunstein I, Cohen-Barak O, Shachaf C, et al: Human telomerase reverse transcriptase promoter regulation in normal and malignant human ovarian epithelial cells. Cancer Res 61: 5529-5536, 2001.

17. Majumdar AS, Hughes DE, Lichtsteiner SP, Wang Z, Lebkowski JS and Vasserot AP: The telomerase reverse transcriptase promoter drives efficacious tumor suicide gene therapy while preventing hepatotoxicity encountered with constitutive promoters. Gene Ther 8: 568-578, 2001.

18. Gu J and Fang B: Telomerase promoter-driven cancer gene therapy. Cancer Biol Ther 2: S64-S70, 2003.

19. Carambula SF, Matikainen T, Lynch MP, et al: Caspase-3 is a pivotal mediator of apoptosis during regression of the ovarian corpus luteum. Endocrinology 143: 1495-1501, 2002.

20. Ninomiya E, Ito Y, Shibata MA, Kawashima K, Sakamoto T, Maruyama E, et al: The activation of caspase-3 and DNA fragmentation in B cells phagocytosed by macrophages. Med Electron Microsc 36: 87-93, 2003.

21. Yudina A and Moonen C: Ultrasound-induced cell permeabilisation and hyperthermia: strategies for local delivery of compounds with intracellular mode of action. Int J Hyperthermia 28: 311-319, 2012.

22. Safinya CR: Structures of lipid-DNA complexes: supramolecular assembly and gene delivery. Curr Opin Struct Biol 11: 440-448, 2001.

23. Vitor MT, Bergami-Santos PC, Barbuto JA, De La and Torre LG: Cationic liposomes as non-viral vector for RNA delivery in cancer immunotherapy. Recent Pat Drug Deliv Formul: Dec 31, 2012 (Epub ahead of print).

24. Feril LB Jr, Kondo T, Zhao QL, et al: Enhancement of ultrasound-induced apoptosis and cell lysis by echo-contrast agents. Ultrasound Med Biol 29: 331-337, 2003.

25. Honda H, Zhao QL and Kondo T: Effects of dissolved gases and an echo contrast agent on apoptosis induced by ultrasound and its mechanism via the mitochondria-caspase pathway. Ultrasound Med Biol 28: 673-682, 2002.

26. Miller MW, Everbach EC, Cox C, Knapp RR, Brayman AA and Sherman TA: A comparison of the hemolytic potential of Optison and Albunex in whole human blood in vitro: acoustic pressure, ultrasound frequency, donor and passive cavitation detection considerations. Ultrasound Med Biol 27: 709-721, 2001.

27 Datiles MJ, Johnson EA and McCarty RE: Inhibition of the ATPase activity of the catalytic portion of ATP synthases by cationic amphiphiles. Biochim Biophys Acta 1777: 362-368, 2008.

28. Krasovitski B, Frenkel V, Shoham $\mathrm{S}$ and Kimmel E: Intramembrane cavitation as a unifying mechanism for ultrasound-induced bioeffects. Proc Natl Acad Sci USA 108: 3258-3263, 2011

29. Zhou Y, Shi J, Cui J and Deng CX: Effects of extracellular calcium on cell membrane resealing in sonoporation. J Control Release 126: 34-43, 2008. 